Military Technical College Kobry El-Kobbah, Cairo, Egypt.



18th International Conference on Applied Mechanics and Mechanical Engineering.

\title{
SIMULATING AN INTERVERTEBRAL LUMBAR SPINE DISC
}

\author{
H. M. Kamel*
}

\begin{abstract}
The intervertebral lumbar spine disc (IVD) is a small oval body that connects between two vertebrae. Its degeneration causes lower back pain, which causes economic losses due to treatment and absence from work. Finite element analysis has been used for understanding the complex degeneration process. However, such studies assumed the disc to behave linearly in the elastic region under static loading. In this work, nonlinear FE analysis was used to model the degeneration process as realistically as possible. This was accomplished using an advanced FE capable of simulating the flow of bodily fluid through the living tissues of the disc. This allowed the material properties to change according to time, load and deformation, which occurs in reality. Furthermore, an innovative approach - DOE - was applied to analysis the effect of changing fluid permeability of the annulus fibrosus (AF) and nucleus pulposus (NP), which are the two main parts of the IVD. The analysis revealed that permeability has a strong effect on the value of deformation of the IVD under load. In addition, there is even a clear interaction effect between the permeability of AF and NP on deformation.
\end{abstract}

\section{KEYWORDS}

Lumbar spine; intervertebral; vertebrate; back pain; finite element

\footnotetext{
* Egyptian Armed Forces.
} 


\section{INTRODUCTION}

The work in this publication is divided into four main parts:

- The anatomy of the lumbar spine and the IVD is introduced to present a background to non-expert readers.

- A concise review of relevant literature in the field of finite element analysis applied to the simulation of the lumbar spine and IVDs.

- A finite element model is developed based on computerized tomography data of a healthy person and the model is validated with published data.

- The Design of experiments approach is applied to examine the effect of change in fluid permeability inside the annulus fibrosus and nucleus pulposus.

Lower back pain can be regarded as a global epidemic. According to the World Health Organization, it is one of the top ten causes of diseases and injuries in the world [1]. In the USA, it is the second most common cause of disability in adults [2]. In the United Kingdom, it was estimated that lower back pain caused the loss of more than 100 million workdays annually [3]. In Germany, the direct cost of treatment is $€$ 7000 per person per year, and lower back pain is the cause in $75 \%$ of the time workers are absent from work [4]. Lower back pain is mostly associated with problems in the intervertebral disc in the spine.

The vertebral spine, also commonly named the backbone, is an amazing structure. It consists of 33 bones, each one is called a vertebrae. As shown in Fig. 1 [5], the spine is divided into five regions, cervical in the neck region (7 vertebrae), thoracic in chest (12), lumbar in the lower back (5), sacral at the base of the spine (5) and finally the coccyx (4). Each vertebra in each group is named by the first letter of the group name, followed by a number, for example, L1 means the first lumbar vertebra. The spine is a multi-functional structure. It supports the head and the trunk, provides attachment links for the limbs, and provides protection for vital organs and the spinal cord. In addition to all these functions, it allows all the human body to perform all sorts of complex and different movements [5].

This is because the spine is flexible and not rigid with 23 intervertebral discs allowing the relative motion between vertebrae. In total length, they are about a quarter of the average $71 \mathrm{~cm}$ long spine. As shown in Fig. 2, the Inter Vertebral Disc (IVD) can be divided in two regions; the inner nucleus pulposus is a gelatinous matter and the outer annulus bulk (fibrosus) made from fibrocartilage. With this multi-material and multi-structure, the IVD cushions and supports loads on the spine. In a standing position, under the weight of the body, IVDs compress and bulge laterally forcing fluid to squeeze out of them. When the body rests, IVDs absorbs back the fluid and they swell. Combined effects lead to the herniated discs, which are injuries to the vertebral spine [5].

Under static, dynamic or fatigue loads, for example lifting heavy weights, violent impact shocks or prolonged wrong postures, cracking occurs in the outer region of the IVD. This causes leakage of the gelatinous nucleus pulposus out. In some cases, this acts pressure on a spinal nerve root or the spinal cord as shown in Fig. 3, which causes pain. The majority of disc herniations ( 95\%) occur in the lumbar region, at levels L4/L5 and L5/S1. At young age, IVDs are well hydrated, but as one gets older, they dehydrate and grow thinner becoming more susceptible to herniation. In general, up to $70 \%$ of the body weight is water during adolescence and 
drops to almost $50 \%$ at old age. This causes stiffening of soft tissue. Bones gradually lose their minerals (demineralization) starting at ages 30-40 years [6].

The relation between intervertebral discs and lower back pain are generally the result of a slow degenerative process. Discs degeneration is a complex process caused by external mechanical loading and internally by discs cells synthesizing their own matrix [8-11]. The study of intervertebral disc degeneration mechanism has spanned many disciplines such as orthopedic surgery, molecular biology and in-vitro simulation [12-14].

The advancement in computer capabilities has promoted the use of finite element method in the study of the degeneration process of intervertebral discs. In1986, Kurowski and Kubo [15] found highest stresses in the center of the healthy discs whereas in degenerated ones, they are located laterally in the cortical wall, and in the vertebral body rims. In 1991, Kim et al. [16] simulated the disc degeneration by removing the hydrostatic capabilities of the nucleus and increasing disc stiffness. In 2002, Goto et al. [17] used computed tomography (CT) images to develop a finite element model of the fourth and fifth lumbar vertebrae. They used their model to study the variation in Von Mises stresses under different loading conditions. In 2006, Rohlmann et al. [18] developed a finite element model of the L3/L4 vertebrae. They modeled degeneration by varying disc height and bulk modulus of the nucleus pulposus. Maren et al. [19] highlighted the capabilities, effectiveness and challenges of the finite element method in studying soft tissues. In a recent study, Lipscomb et al. [20] conducted an experimental program and a FE simulation to investigate whether discography, which has been used to diagnose the conditions of discs is itself cause disc degeneration. Their findings proved this hypothesis.

Despite the critical role, the IVD plays in the development of lower back pain; its behavior under constant stress has not been thoroughly examined. Moreover, in the limited work that has been published, the problem has been simplified as linearly static problem [21-23], where in reality, materials properties of the IVD vary with load and time [24, 25].

In the present work, ANSYS finite element package was used to simulate the intervertebral lumbar spine disc under compressive load. The coupled pore-pressure element capabilities in the software modeled the IVD and simulated its behavior under creep loading.

\section{FINITE ELEMENT SIMULATION}

The model geometry is shown in Fig. 4; it shows the intervertebral disc of the lumbar spine between the fourth and fifth vertebrae. It represents a healthy human adult subject.

The disc was meshed using the CPT217 element. The CPT217 is a 3-D 10-node element, which has elasticity, stress stiffening, large deflection, and large strain capabilities. It is suitable for modeling multi-phase systems, where a fluid passes through a porous media. This makes the CPT217 element an ideal selection for modelling the flow of fluid through the outer annulus of the IVD under compression load of the lumbar spine. The lower vertebrae bones were meshed using the 
SOLID187 element. The total number of elements is 36538 and number of nodes is 90000. Material properties required as input to the FE model are given in Table 1 [26].

The nodes of the lower surface vertebrae were fixed in all directions and a load of $500 \mathrm{~N}$ was applied at the center node at the top surface of the upper vertebrae. The model was solved using nonlinear static analysis in ANSYS to represent creep during a five days period (4.27E5 sec.).

\section{Model Validation}

The model was developed in ANSYS and was run on an i7 8 GB RAM laptop running Windows 64 bit, which allowed the extension of the physical memory even more. The simulation lasted for approximately ten hours, which showed the extent of complexity of the problem.

The model was verified using the simulation results that have been published by ANSYS Inc. [27]. The report examines the simulation results of an IVD during a period of five days [27]. The Von-Mises stress distribution around the IVD is in very good agreement with published results as shown in Fig. 6 . The difference between maximum values is $13.5 \%$. The longitudinal deformation is identical as shown in Fig. 7.

\section{RESULTS AND DISCUSSIONS}

Figure 5 shows the distribution of deformation over the IVD, upper and lower vertebrae. It shows the bulging of the IVD under compressive loading after creep. This will force the fluid out of the IVD from the inner nucleus pulposus through the outer annulus.

Looking more closely on the stress distribution over the IVD only as shown in Fig. 6, relatively higher stresses have been developed over the outer area with a maximum value at one outer edge. These areas of high stress values are susceptible to failure especially after enough fatigue loading. After which, cracks will develop at the outer annulus causing leakage of fluid out of the IVD. This will lead to stiffening of the IVD, gradually losing its ability to cushion loads between the two vertebrae. This shows how the mechanism of lower back pain develops.

Figure 7 shows the deformation in the vertical direction of the center node of the IVD over the loading period of five days. It shows that at the onset of load application, the inner of the IVD deformed about $0.4 \mathrm{~mm}$. Then, it converged to approximately $1.5 \mathrm{~mm}$ after one and half days. The deformation response as presented in Fig. 8 shows the development of normal stress on the upper surface of the IVD. It also shows that the stress converges approximately $0.9 \mathrm{MPa}$ after the same one and half days' period.

Assuming that twelve hours is the maximum number a worker normally works. Therefore, during rest, a regenerative process works to replenish the IVD with some or all the fluid that has been squeezed out under loading. This will restore some or all of the elasticity of the IVD. However, aging works to decrease the amount of fluid that 
is already in the cells, moreover, fatigue and extreme loading will also promote the degeneration of the IVD.

The degenerative process of the IVD can be slowed down by following some known advices such as:

- Exercise to strengthen the muscles of the back to help in reducing the load on the IVD.

- Losing weight, especially belly fat that shifts the center of gravity of the body forward posing a constant bending moment on the spine.

- Avoiding sudden and harsh movements of the spine.

- Special care when lifting heavy objects.

\section{Effect of Permeability}

The effect of permeability on the total deformation of the IVD is investigated using the validated FE model. According to literature, permeability of the annulus fibrosus (AF) and nucleus pulposus (NP) vary widely according to the testing methods and human individual variabilities. Minimum and maximum values of permeability for AF and NP were collected from literature [28]. These values represent the two levels between permeability changes. They were then used to form a matrix representing all the combinations as listed in Table 2, in addition to the values, which were previously used in developing the FE model. The values are then used to simulate the IVD under the same load during a twelve hours' time. The total deformation is calculated for each case as shown in Table 2.

The presented results in Table 2 are analyzed using the Design of Experiments (DOE) approach, which is a statistical technique used to maximize information gain with minimum number of experiments. In this work, we used all combinations, which is named, a full factorial approach. Two useful information are derived from the analysis of the date.

Figure 9 shows the main effect of changing permeability from low to high values. It is clear the permeability of AF and NP has a pronounced positive effect on the total deformation of the IVD. Increasing permeability increases total deformation.

Figure 10 shows the interaction effect between the change in permeability values of $\mathrm{AF}$ and NP on total deformation. It is interesting to note that for low permeability values of $\mathrm{AF}$, there is a noticeable effect on total deformation. When the permeability of the NP changes from low (3) to high (33.5), total deformation increases from $\sim 1.4$ $\mathrm{mm}$ to $\sim 1.6 \mathrm{~mm}$.

This shows that the permeability of the AF and NP each has a strong effect on the total deformation of the IVD. In addition, the analysis revealed that there an interaction between the two. To the author's best knowledge, this observation has not been presented before. The factors causing permeability to change are numerous: nutrition, age, physical load ... etc. This is the subject of further discussion and analysis in future work. 


\section{CONCLUSIONS}

The finite element method was used to model and simulate the response of the intervertebral lumbar spine disc under axial loading for a prolonged duration of time. The results showed the working mechanism of the disc degenerative process. We succeeded in modeling the fluid interaction between the solid vertebrae and the porous intervertebral disc. The results showed that under compressive loading, stress reaches a constant value after approximately one and a half days. Although this is an exceptionally long period, however, we can conclude that extended loading of the lumbar spine should be avoided. In addition, exercise, diet and avoidance of sudden and harsh movements are recommended to slow the degeneration of the disc.

An innovative statistical technique - DOE - was used to reveal main and interaction effects of permeability values of annulus fibrosus and nucleus pulposus on the total deformation of the intervertebral disc. The physiological causes of the change in permeability is left for future analysis.

\section{REFERENCES}

[1] $\mathrm{Ng}$, Marie, et al., "Global, regional, and national prevalence of overweight and obesity in children and adults during 1980--2013: a systematic analysis for the Global Burden of Disease Study 2013", The Lancet, 384(9945): p. 766-781 (2014).

[2] Centers for Disease Control Prevention, CDC, "Prevalence of disabilities and associated health conditions among adults--United States, 1999", MMWR. Morbidity and mortality weekly report, 50(7): p. 120 (2001).

[3] Papageorgiou, A. C., et al., "Influence of previous pain experience on the episode incidence of low back pain: results from the South Manchester Back Pain Study", Pain, 66(2-3): p. 2-3 (1996).

[4] Juniper, Melissa, et al., "The epidemiology, economic burden, and pharmacological treatment of chronic low back pain in France, Germany, Italy, Spain and the UK: a literature-based review", Expert Opinion on Pharmacotherapy, 10(16): p. 2581-2592 (2009).

[5] Saladin, Kenneth S., "Human anatomy", McGraw-Hill. p. 151-182 (2014),

[6] Kai-Uwe Schmitt, et al., "Trauma Biomechanics, An Introduction to Injury Biomechanics", Springer-Verlag Berlin Heidelberg (2014).

[7] Netter, Frank H., "Atlas of human anatomy", Saunders/Elsevier. p. 152-159 (2011).

[8] Adams, Michael A., et al., "Mechanical Initiation of Intervertebral Disc Degeneration". Spine, 25(13) (2000).

[9] Stokes, Ian A. F., et al., "Mechanical conditions that accelerate intervertebral disc degeneration: overload versus immobilization". Spine, 29(23): p. 27242732 (2004).

[10] Adams, M. A., et al., "The effect of posture on the fluid content of lumbar intervertebral discs", Spine, 8(6): p. 665-671 (1983).

[11] Buckwalter, Joseph A., "Aging and degeneration of the human intervertebral disc". Spine, 20(11): p. 1307-1314 (1995). 
[12] Toyone, T., et al., "Vertebral bone-marrow changes in degenerative lumbar disc disease. An MRI study of 74 patients with low back pain", Bone \& Joint Journal, 76(5): p. 757-764 (1994).

[13] Walsh, Andrew J.L., et al., "In vivo growth factor treatment of degenerated intervertebral discs", Spine, 29(2): p. 156-163 (2004).

[14] Adams, Michael A., et al., "What is Intervertebral Disc Degeneration, and What Causes It?", Spine, 31(18) (2006).

[15] Kurowski, Pawel, et al., "The relationship of degeneration of the intervertebral disc to mechanical loading conditions on lumbar vertebrae", Spine, 11(7): p. 726-731 (1986).

[16] Kim, Younge, et al., "Effect of disc degeneration at one level on the adjacent level in axial mode". Spine, 16(3): p. 331-335 (1991).

[17] Goto, Keisuke, et al., "Mechanical analysis of the lumbar vertebrae in a threedimensional finite element method model in which intradiscal pressure in the nucleus pulposus was used to establish the model", Journal of Orthopaedic Science, 7(2): p. 243-246 (2002).

[18] Rohlmann, Antonius, et al., "Analysis of the influence of disc degeneration on the mechanical behaviour of a lumbar motion segment using the finite element method", Journal of Biomechanics, 39(13): p. 2484-2490 (2006).

[19] Freutel, Maren, et al., "Finite element modeling of soft tissues: material models, tissue interaction and challenges", Clinical Biomechanics, 29(4): p. 363-372 (2014).

[20. Lipscomb, K. E., et al., "Biomechanical Effects of Human Lumbar Discography: In Vitro Experiments and Their Finite Element Validation", Clinical spine surgery, 30(3): p. 219-219 (2017).

[21] Kitazaki, S., et al., "A modal analysis of whole-body vertical vibration, using a finite element model of the human body", Journal of Sound and Vibration, 200(1): p. 83-103 (1997).

[22] Van Toen, Carolyn, et al., "Transmission of Force in the Lumbosacral Spine During Backward Falls", Spine, 37(9): p. E519-E527 (2012).

[23] Gan, Zengkang, et al., "Biodynamic modelling of seated human subjects exposed to uncouples vertical and fore-and-aft whole-body vibration", Journal of Vibration Engineering and Technologies, 3(3): p. 301-314 (2015).

[24] Marini, Giacomo, et al., "Nonlinear dynamics of the human lumbar intervertebral disc", Journal of Biomechanics. 48(3): p. 479-488.

[25] Marini, Giacomo, et al., "A 1-D model of the nonlinear dynamics of the human lumbar intervertebral disc", Journal of Sound and Vibration, 387(Supplement C): p. 194-206 (2017).

[26] Argoubi, M, et al., "Poroelastic creep response analysis of a lumbar motion segment in compression", Journal of Biomechanics, 29(10): p. 1331-1339 (1996).

[27] IP, SAS, "Simulation of a Lumbar Motion Segment", in ANSYS Mechanical APDL Technology Demonstration Guide. South pointe, 2600 ANSYS Drive. (2017). 


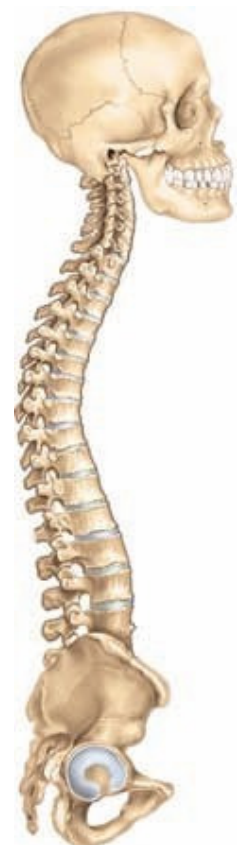

(a)

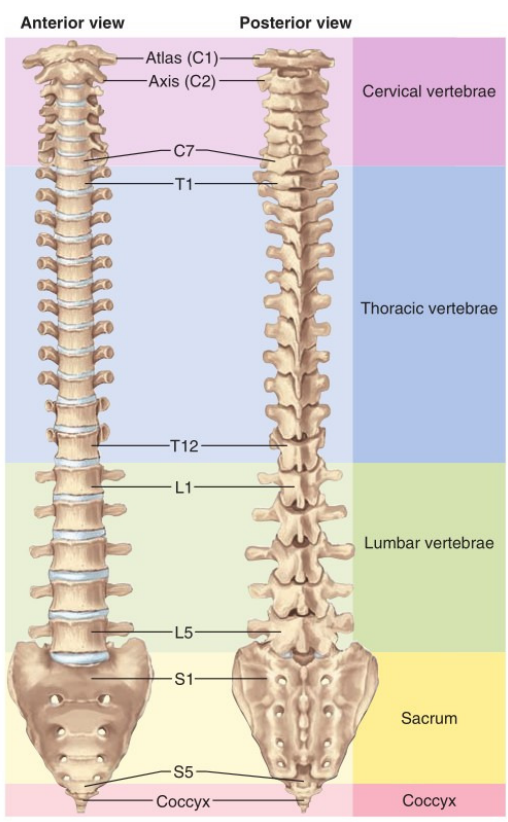

(b)

Fig. 1. (a) The human spine. (b) The vertebral spine [5].

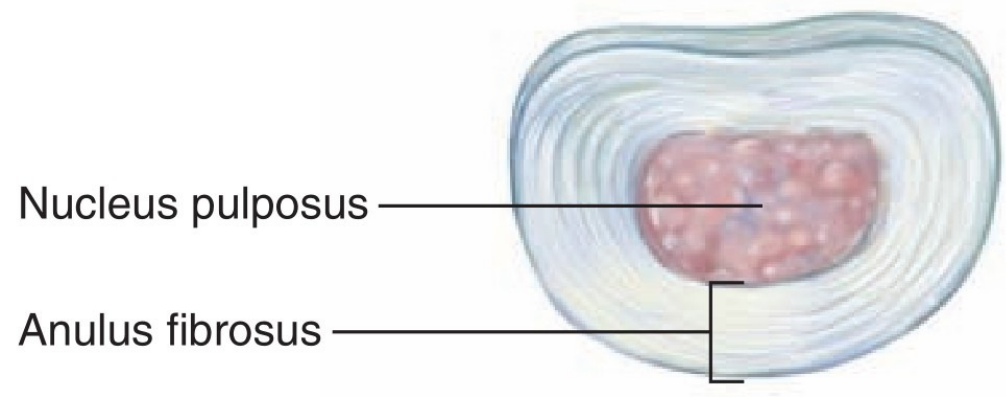

Fig. 2. The intervertebral disc [5].

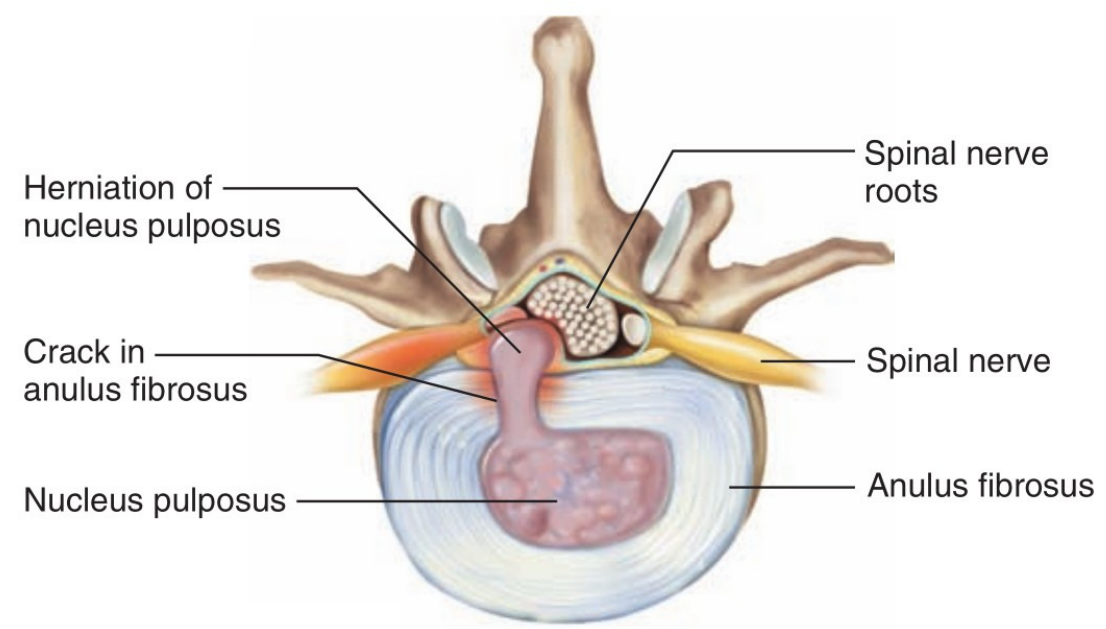

Fig. 3. Herniated disc [7]. 


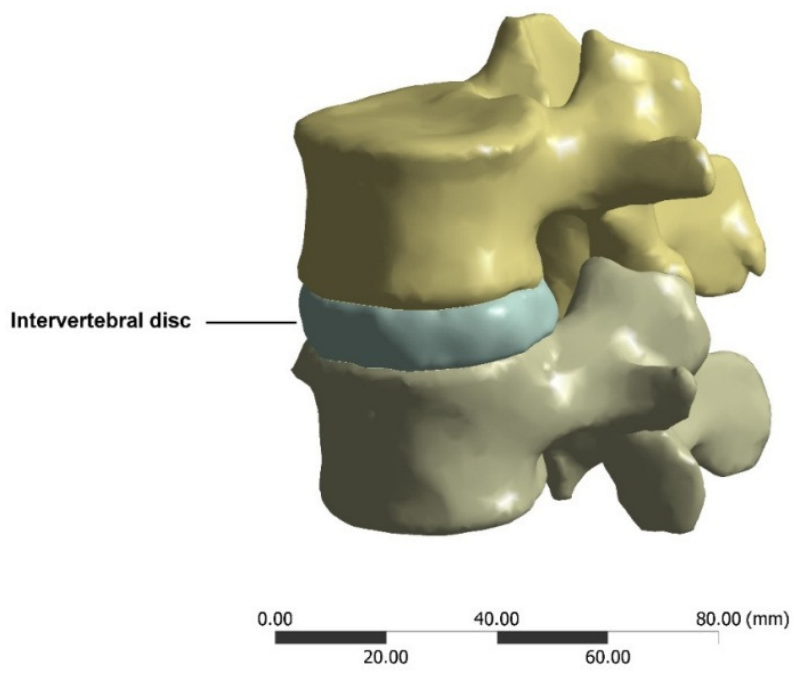

Fig. 4. Geometry of the IVD.


Fig. 5. Distribution of deformation.

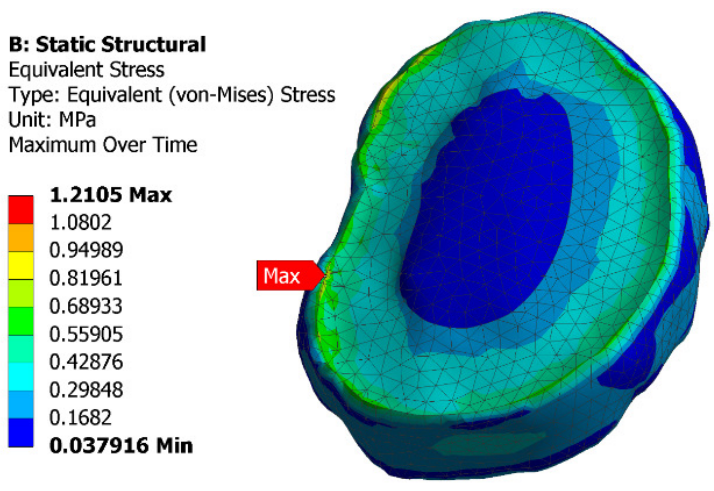

(a) Simulated FE results.

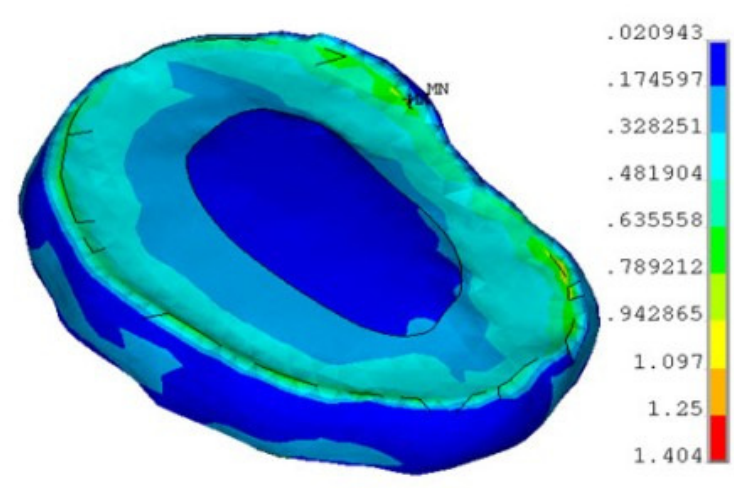

(b) Published results [27].

Fig. 6. Distribution of von-Mises stress over the IVD. 


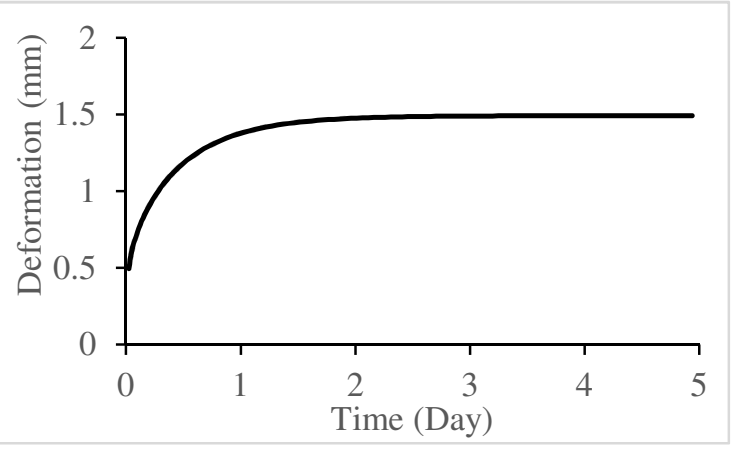

(a) Simulated FE results

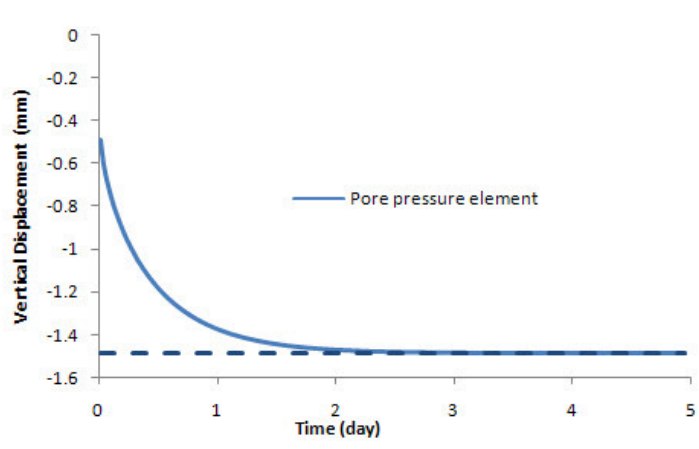

(b) Published results [27]

Fig. 7. Deformation of the IVD over time.

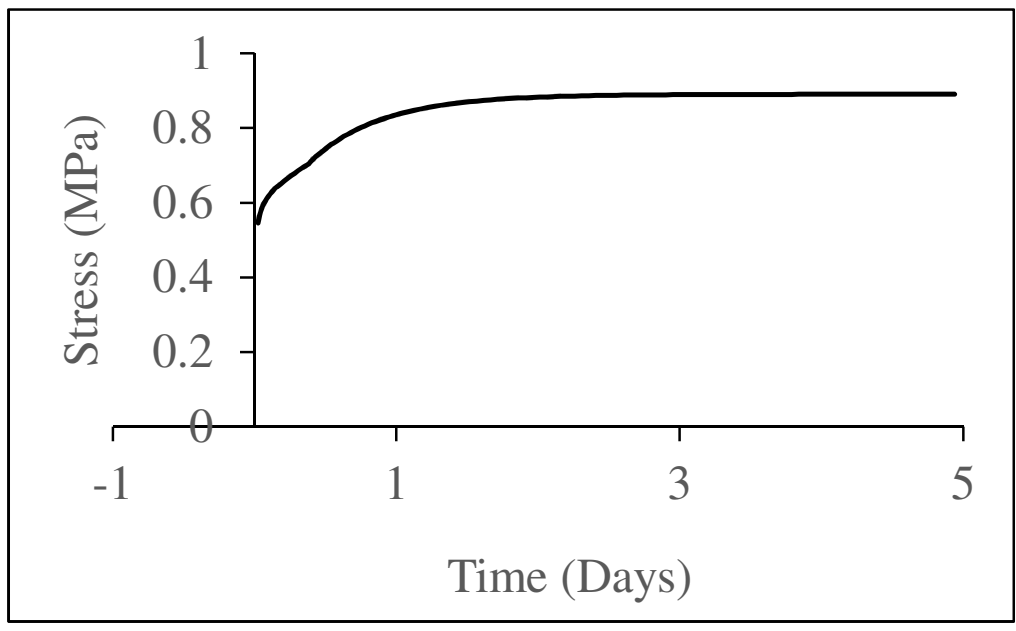

Fig. 8. Normal stress on the upper surface of the IVD over time.



Fig. 9. Main effect of permeability on deformation. 


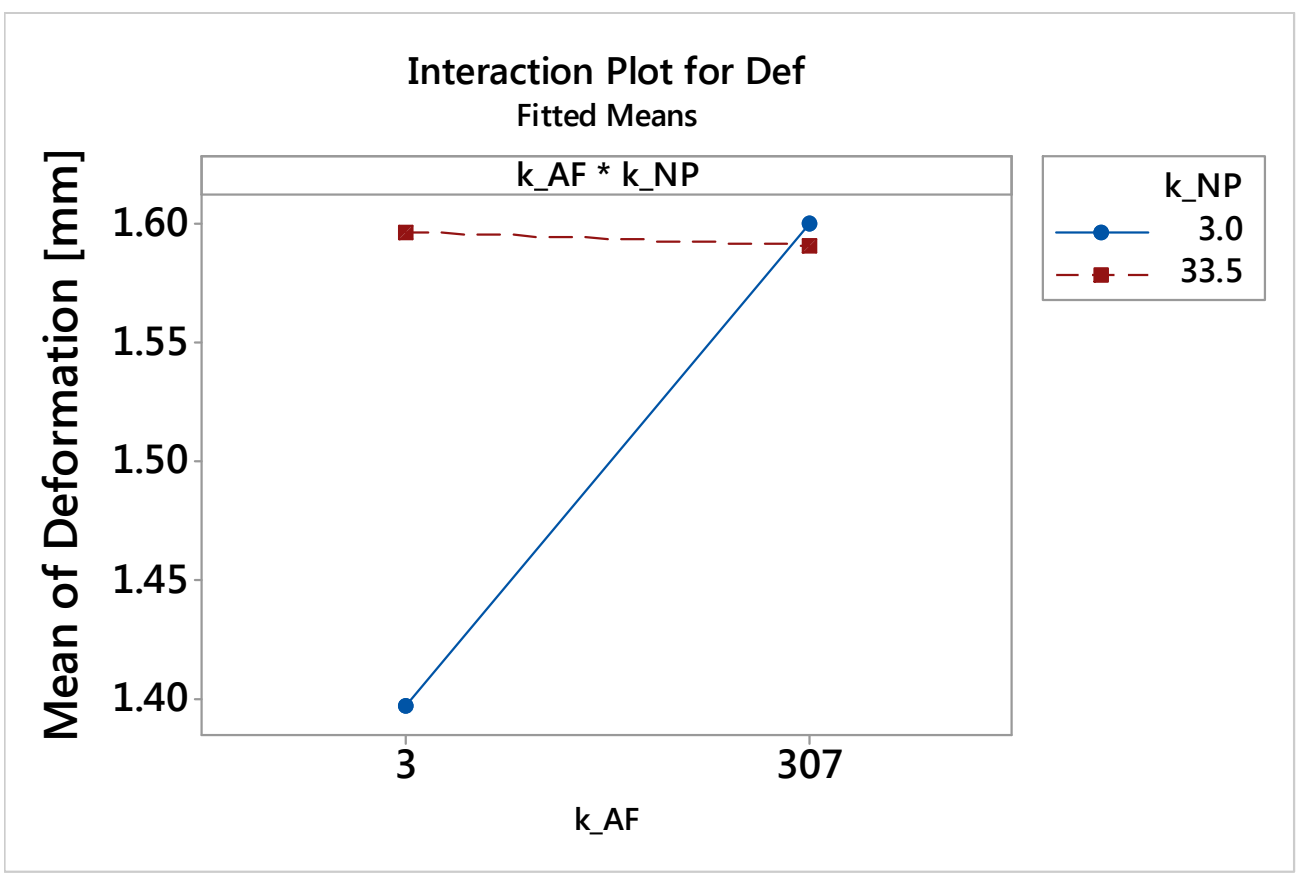

Fig. 10. Interaction effect of permeability on deformation.

Table 1. Material properties of the IVD [26].

\begin{tabular}{|l|c|c|c|}
\hline \multicolumn{1}{|c|}{ Property } & $\begin{array}{c}\text { Annulus } \\
\text { Bulk }\end{array}$ & $\begin{array}{c}\text { Nucleus } \\
\text { Pulposus }\end{array}$ & Bone \\
\hline Elastic Modulus, (MPa) & 2.5 & 1.5 & 3500 \\
\hline Poisson's Ratio & 0.10 & 0.1 & 0.2 \\
\hline Permeability (m-1·s-1) $\times 10-16$ & 3 & 3 & - \\
\hline $\begin{array}{l}\text { Yield Strength in } \\
\text { compression, (MPa) }\end{array}$ & - & - & 6 \\
\hline $\begin{array}{l}\text { Ultimate Strength in } \\
\text { compression, (MPa) }\end{array}$ & - & - & 11 \\
\hline
\end{tabular}

Table 2. Values of permeability and simulated deformation.

\begin{tabular}{|c|c|c|}
\hline \multicolumn{2}{|c|}{ Permeability (m-1.5-1) $\mathbf{\times 1 0 - 1 6}$} & \multirow{2}{*}{ Deformation (mm) } \\
\hline AF & NP & \\
\hline 53 & 3.9 & 1.571 \\
\hline 307 & 3.9 & 1.578 \\
\hline 53 & 33.5 & 1.592 \\
\hline 307 & 33.5 & 1.592 \\
\hline 3 & 3.0 & 1.287 \\
\hline
\end{tabular}

